\title{
Kappa light-chain amyloidosis of gastrointestinal tract
}

\author{
Rintaro Hashimoto ${ }^{a, b}$, Tomoki Matsuda ${ }^{b}$ \\ University of California, Irvine; Sendai Kousei Hospital, Japan
}

\begin{abstract}
A 65-year-old man with no previous medical history presented to our hospital with hematochezia. The patient's vital signs were normal. Physical examination revealed nothing remarkable except left lower quadrant tenderness. Laboratory data indicated mild anemia. Upper endoscopy revealed small erosion in the esophagus (Fig. 1A), mild linear erythema in the lesser curvatures of the gastric body (Fig. 1B), and normal appearance of the mucosa in the duodenum. Colonoscopy revealed multiple submucosal hematomas (Fig. 1C) and ulcerations (Fig. 1D). Biopsies were taken from the stomach and the colonic lesions. Histopathological examination with Congo red staining revealed amorphous eosinophilic infiltrates, while immunostaining revealed found the cells to be positive for kappa light chain (Fig. 2) and negative for lambda chain and amyloid A. The patient was diagnosed with systemic kappa amyloid light-chain (AL) amyloidosis given the involvement of heart and kidney. The patient was transferred to the hematology department and received chemotherapy.

Amyloidosis is characterized by the extracellular deposition of abnormal fibrillary protein. The endoscopic findings have been reported to be very diverse but characteristic [1]. The treatment for $\mathrm{AL}$ amyloidosis with evidence of organ involvement is chemotherapy and autologous stem cell transplantation [2]. In our case, although the clinical course at the first episode was compatible with ischemic colitis, retrospective evaluation of biopsy specimens from the first episode revealed they were positive for Congo red staining. Amyloidosis should be considered in patients with non-specific ulcerative findings and Congo red staining should be performed as part of the histopathological evaluation.
\end{abstract}

aDivision of Gastroenterology, University of California, Irvine;

bDepartment of Gastroenterology, Sendai Kousei Hospital, Japan

Conflict of Interest: None

Correspondence to: Rintaro Hashimoto, Division of Gastroenterology, University of California, Irvine, 101 The City Drive, Bldg. 22C, Orange, CA 92868, USA, e-mail: rintaroh@uci.edu

Received 6 September 2018; accepted 16 September 2018; published online 3 October 2018

DOI: https://doi.org/10.20524/aog.2018.0319

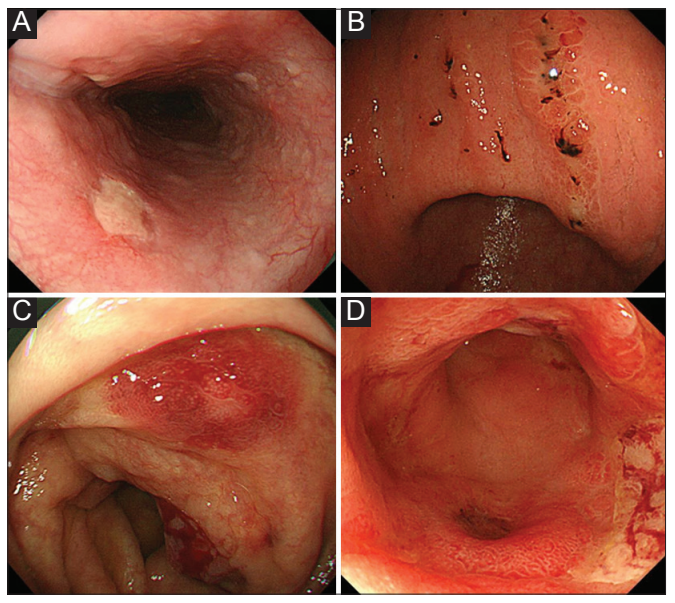

Figure 1 Upper endoscopy showing (A) small erosion in the esophagus and (B) mild linear erythema in the stomach. Colonoscopy showing (C) submucosal hematoma and (D) ulcerations

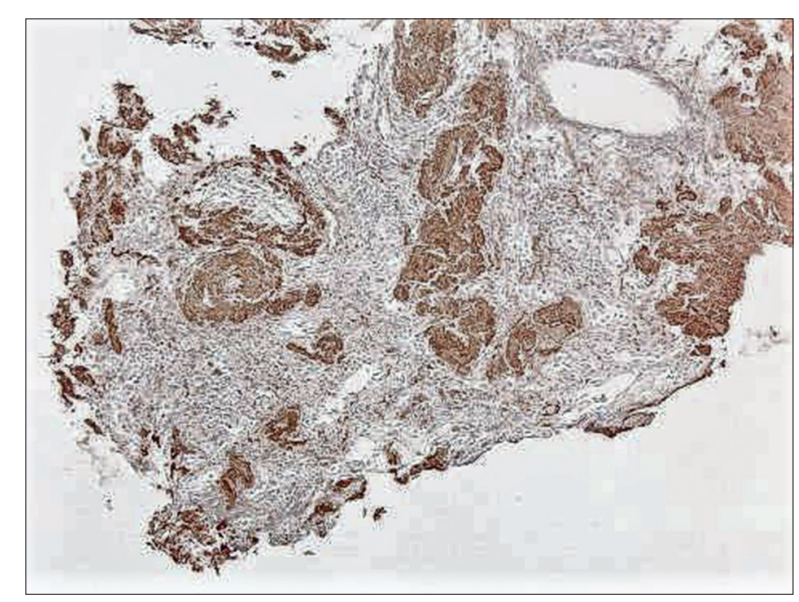

Figure 2 Congo red staining showing amyloid deposition

\section{References}

1. Iida $T$, Yamano $H$, Nakase $H$. Systemic amyloidosis with gastrointestinal involvement: diagnosis from endoscopic and histological views. J Gastroenterol Hepatol 2018;33:583-590.

2. Agis H. News in AL Amyloidosis ASH 2016: A rapidly evolving field of investigation. Memo 2017;10:66-71. 